\title{
CYTOHISTOPATHOLOGICAL CORRELATION OF NEOPLASTIC AND NON-NEOPLASTIC LESIONS OF LYMPH NODE- A DIAGNOSTIC TEST VALIDATION STUDY
}

\author{
Sheela K. Mㄴ, Priya M. G
}

${ }^{1}$ Assistant Professor, Department of Pathology, Government Medical College, Thiruvananthapuram. ${ }^{2}$ Assistant Professor, Department of Pathology, Government Medical College, Thiruvananthapuram.

\section{ABSTRACT}

\section{BACKGROUND}

Fine needle aspiration cytology is a simple and cost effective technique that is routinely used in patients presenting with persistent lymphadenopathy. FNAC is a first line investigation modality for evaluation of lymphadenopathy. Aetiology of lymphadenopathy in head and neck region varies from reactive hyperplasia to tuberculous granulomatous lesion to malignancy.

The aim of the present study is to compare the accuracy of FNAC in the diagnosis of neoplastic lymph node disease with that of non-neoplastic diseases.

\section{MATERIALS AND METHODS}

This descriptive retrospective study was undertaken at Pathology Department, Government Medical College, Thiruvananthapuram over a period of two years. Out of 1020 lymph node aspirations received in our cytology department, a total of 122 cases were identified and included in this study where both cytology and biopsy material were available for review. All the diagnosis obtained by fine needle aspiration cytology was correlated with histopathology.

\section{RESULTS}

Out of 122 lesions in this study, 77 cases were non-neoplastic and 45 cases were neoplastic. The best diagnostic accuracy on cytohistopathological correlation was in cases of metastatic carcinoma (89\%) followed by Non-Hodgkin's lymphoma (88.8\%), reactive hyperplasia (79.2\%) and tuberculosis lymphadenitis $(71.4 \%)$ respectively. The overall accuracy of non-neoplastic lymph node lesions by FNA was found to be $91.8 \%$, sensitivity $99.2 \%$ and specificity $84.3 \%$. In neoplastic lymph node lesions of FNA, the overall accuracy was found to be $98.4 \%$, sensitivity $100 \%$ and specificity $97.5 \%$

\section{CONCLUSION}

FNAC is an important diagnostic tool for diagnosing benign as well as malignant lesions. Findings in this study are comparable and consistent with studies conducted elsewhere. In the present study, it is observed that the accuracy of FNAC is marginally more in diagnosing neoplastic nodal diseases than non-neoplastic nodal diseases.

\section{KEYWORDS}

FNAC, Lymphadenopathy, Lymphoma, Tuberculous Lymphadenitis, Neoplastic Lymph Node Lesions, Non-neoplastic Lymph Node Lesions.

HOW TO CITE THIS ARTICLE: Sheela KM, Priya MG. Cytohistopathological correlation of neoplastic and non-neoplastic lesions of lymph node- A diagnostic test validation study. J. Evolution Med. Dent. Sci. 2017;6(45):3519-3523, DOI: $10.14260 /$ Jemds/2017/759

\section{BACKGROUND}

FNAC is an accurate diagnostic technique in diagnosing aetiology of lymphadenopathies. It is a simple and easy technique in evaluating lymph node lesions because of their superficial locations and easy accessibility.[1]

Aspiration of lymph nodes for diagnostic purpose was reported as early as 1904 by Greig and Gray who used this procedure in the diagnosis of trypanosomiasis.[2],[3]

This technique is a simple, quick and cost effective method to sample superficial masses like cervical lymph node. Lymphadenopathy is one of the commonest symptoms of all age groups attending outpatient department. Causes of peripheral lymphadenopathy include reactive hyperplasia of

Financial or Other, Competing Interest: None.

Submission 29-04-2017, Peer Review 20-05-2017,

Acceptance 29-05-2017, Published 05-06-2017.

Corresponding Author:

Dr. Sheela K. M,

Samanuaya, STRA-33,

Thuruvikkal (P. O.), Ulloor,

Thiruvananthapuram-695011, Kerala.

E-mail: sheelakm51@gmail.com

DOI: $10.14260 /$ jemds $/ 2017 / 759$ lymph node, tuberculous lymphadenitis, malignant diseases like Non-Hodgkin's lymphoma, Hodgkin's lymphoma and metastatic diseases. ${ }^{[4]}$ FNAC has gained considerable importance in patients presenting with Lymphadenopathy.

FNAC has been a suitable investigation to rule out malignancies and to confirm reactive or infective pathology. [5] It has emerged as an advanced diagnostic tool to differentiate reactive hyperplasia/inflammatory conditions, granulomatous disorders and lymphomas.

The objective of our study was to compare the accuracy of FNAC in the diagnosis of neoplastic lymph node disease with that of non-neoplastic diseases.

\section{MATERIALS AND METHODS}

This descriptive retrospective study was undertaken at Pathology Department, Government Medical College, Thiruvananthapuram over a period of two years. Out of 1020 lymph node aspirations received in our cytology department, a total of 122 cases were identified and included in the study where both cytology and biopsy materials were available for review. The cases were collected at random with no preference for age, sex or any other criteria. FNAC of the 
lymph node lesions was compared with histopathology. This study was approved by the ethics committee of the institution.

Aspiration of the lymph nodes was done under aseptic precautions using 22/23 gauge needle and $10 \mathrm{~mL}$ syringe. A minimum of 2 to 3 smears were taken out of which one was air dried for Giemsa staining when required and others were fixed in 95\% ethyl alcohol for Papanicolaou staining. The histopathology of the excision biopsy specimen was studied and final histopathology report was compared with FNAC report to assess the accuracy of cytodiagnosis. According to the proforma developed for the study all data were entered. Statistical analysis was done using Epi-Info software. A positive cytohistopathological correlation was taken as true positive (TP) whereas cytohistopathological disagreement was either false positive (FP) that is cytology positive and histology negative or false negative (FN) that is cytology negative and histopathology negative. Cases where cytological smear were negative and corresponding biopsies also did not reveal any pathological lesion were taken as true negative. Histopathology was the gold standard.

\section{RESULTS}

Out of 122 lesions in this study, 77 cases were nonneoplastic and 45 cases were neoplastic. Among the nonneoplastic lymph node lesions, the commonest was reactive change (52\%) followed by tuberculous lymphadenitis (34\%), necrotising lymphadenitis $(6 \%)$, suppurative lymphadenitis (6\%), eosinophilic lymphadenitis (1\%) and toxoplasma lymphadenitis (1\%) respectively.
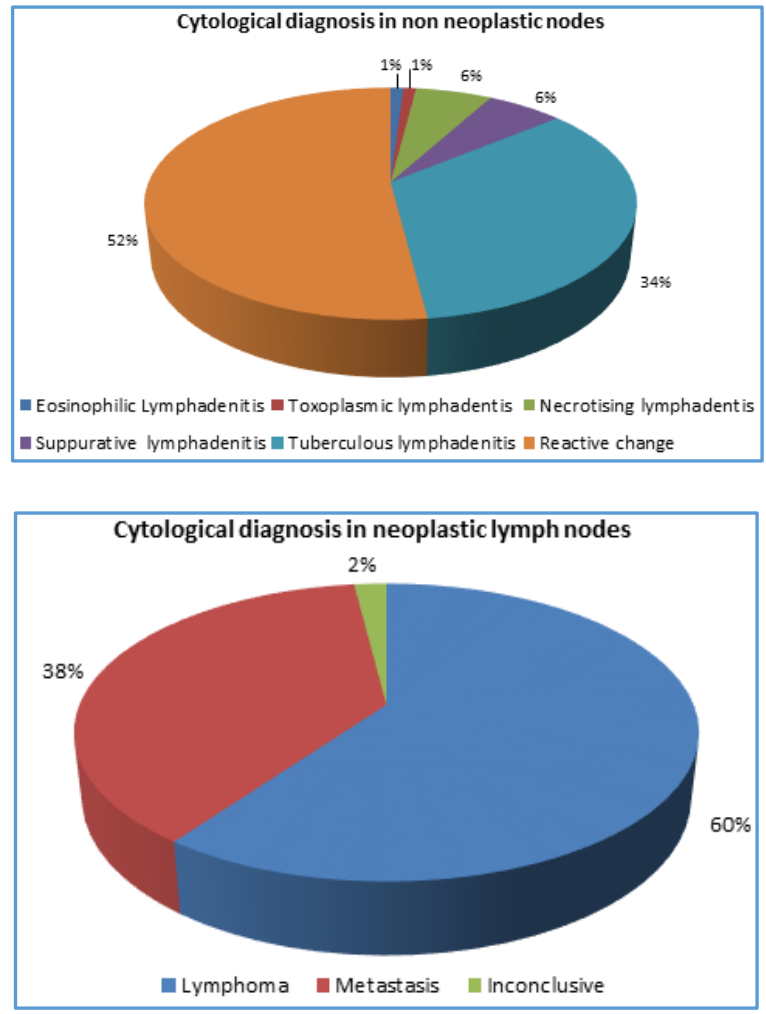

Lymphoma was commonest among neoplastic lesions (60\%) followed by metastasis (38\%).

Histology of non-neoplastic lymph node lesions revealed tuberculous lymphadenitis was the commonest (59\%) followed by reactive change (40\%). Among the histopathological diagnosis of neoplastic lymph nodes, the commonest was lymphoma $(60 \%)$ followed by metastasis (38\%) and plasmacytoma (2\%).
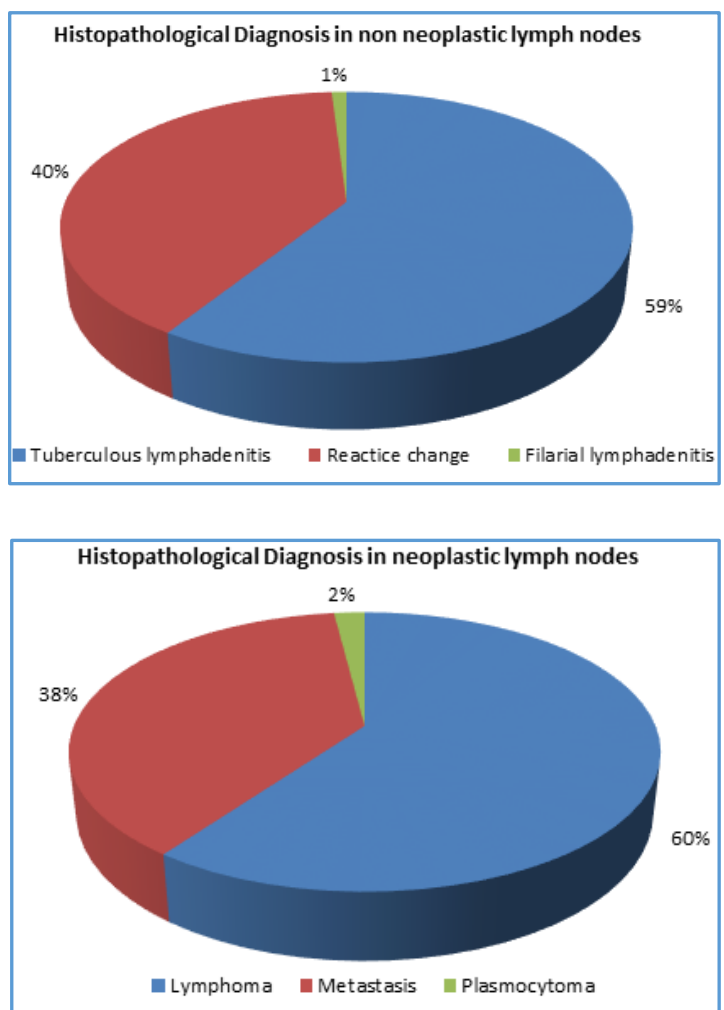

In non-neoplastic lymph node lesions by FNAC, the overall accuracy was $91.8 \%$, sensitivity $97.2 \%$ and specificity 84.3\%. In neoplastic lymph node lesions by FNAC, the overall accuracy was found to be $98.4 \%$, sensitivity $100 \%$ and specificity $97.5 \%$.

Comparison of Statistical Parameters of Neoplastic and Non-Neoplastic Lymph node lesions by FNAC

\begin{tabular}{|c|c|c|}
\hline Diagnostic Indices & $\begin{array}{c}\text { Non-Neoplastic } \\
\text { Lesions }\end{array}$ & $\begin{array}{c}\text { Neoplastic } \\
\text { Lesions }\end{array}$ \\
\hline Sensitivity & $97.2 \%$ & $100 \%$ \\
\hline Specificity & $84.3 \%$ & $97.5 \%$ \\
\hline Positive predictive value & $89.6 \%$ & $95.45 \%$ \\
\hline Negative predictive value & $95.56 \%$ & $100 \%$ \\
\hline Overall accuracy & $91.8 \%$ & $98.4 \%$ \\
\hline
\end{tabular}

\section{DISCUSSION}

Lymphadenopathy is a clinical manifestation of local and systemic diseases which serves as an excellent clue to the underlying disease process. FNAC of lymph node serves as an excellent and rapid first line investigation modality to establish the nature of lesion.[6] It is a simple technique in the diagnosis of lymphadenopathies which not only offers tissue diagnosis but also serves as a preliminary screening procedure for a number of clinical considerations like lymphoma, metastasis, tuberculosis, etc. The high specificity of techniques helps to single out those that need further investigation or biopsy and obviates the need for unnecessary surgical excision of nodes. We conducted a retrospective study which included 122 cases of lymphadenopathy to compare the accuracy of FNAC in the diagnosis of neoplastic 
lymph node diseases with that of non-neoplastic lymph node diseases.

Reactive change was the commonest forming $32 \%$ of total lymph node lesions; the sensitivity was $89 \%$ and specificity $72 \%$. Barbara L Steel et al in her studies had concluded that the ability to distinguish reactive process and lymphoma cytologically is significantly lower.[7]

In our study, there were 26 cases of tuberculous lymphadenitis which constituted $20.49 \%$ of lymph node lesions. The diagnostic accuracy of tuberculous lymphadenitis was $71 \%$ with low sensitivity (54\%) and high specificity (91\%). Several of the cases of tuberculous adenitis were diagnosed as necrotising adenitis due to the presence of necrotic material and nuclear debris. Pablo Lapuerta et al in their studies have remarked that the most reliable criterion for tuberculous lymphadenitis are the presence of stainable AFB and culture of organisms from the aspirate. ${ }^{[8]}$ Handa $U$ et al in their work had concluded that FNA of tuberculous lymphadenopathy provided a high level of diagnostic accuracy and that FNA is reliable as an initial evaluating procedure for diagnosis of tuberculous lymphadenitis.[9]

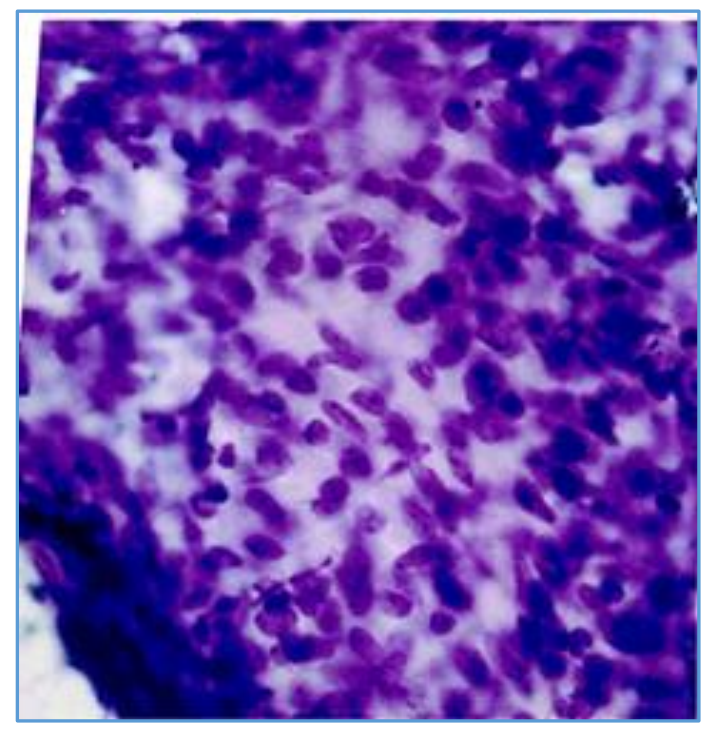

Figure 1. FNAC of Lymph Node Showing Epithelioid Cells

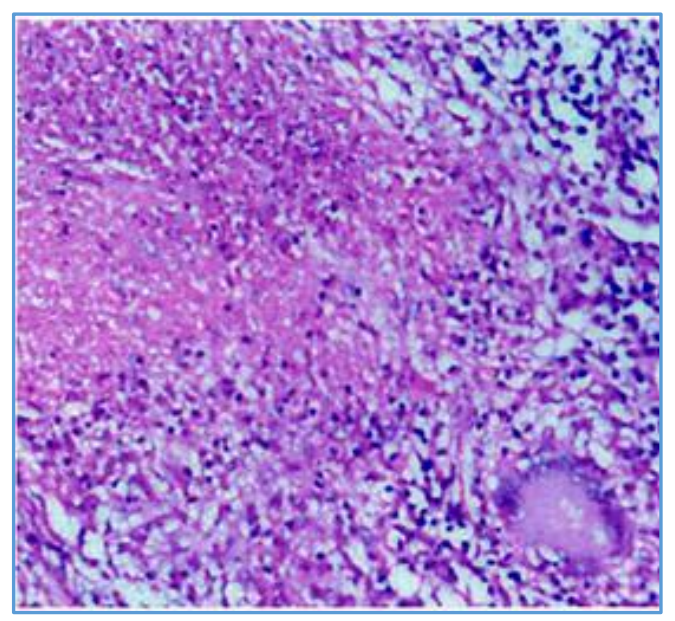

Figure 2. Histopathology of Caseating Granulomatous Lymphadenitis Showing Caseation, Epithelioid Cells and Langhans' Giant Cell
Necrotising lymphadenitis constituted $4.1 \%$ of total lymph node lesions. Out of five cases of necrotising lymphadenitis, four cases were diagnosed as caseating granulomatous lesions consistent with tuberculosis. In one case, histopathology showed Hodgkin's Lymphoma, Nodular Sclerosis. AFB staining should be done in all cases where necrosis is seen.

Suppurative lymphadenitis constituted five cases and $4.1 \%$ of total lymph node lesions. In all the cases, histopathology showed caseating granulomatous adenitis consistent with tuberculosis. This observation clearly suggests that when necrosis is seen with or without neutrophils, tuberculosis should be considered and epithelioid cells looked for. AFB staining in these cases may be useful.

Eosinophilic lymphadenitis constituted only one case in our study which on histopathology showed reactive change. Only one case of toxoplasma lymphadenitis could be found in our study which on biopsy showed caseating granulomatous lesion consistent with tuberculosis. Caseous necrosis was absent in FNAC. So whenever epithelioid cell collections are seen without caseous necrosis serology for toxoplasma should be done.

During the study period, 45 cases of neoplastic lesions were included which constituted $36.88 \%$ of total lymph node lesions. This group included malignant lymphoma and metastatic lesions. A total of 27 cases of malignant lymphoma (22.13\%) were studied during this period which included 23 cases of Non-Hodgkin's Lymphoma and 4 cases of Hodgkin's Lymphoma. Among 23 cases of NHL, 18 cases were correctly diagnosed. One case of NHL turned out to be Plasmacytoma on histopathology. Mathur et al in his studies had concluded that in spite of limitations inherent to the procedure FNAC is a useful diagnostic tool for diagnosis of large majority of cases and this can be used to separate NHL into clinically relevant grades to predict diagnosis.[10] The diagnostic accuracy of NHL in this study was $88.88 \%$. Naeem Ahmed et al have suggested that the use of FNAC to diagnose lymphoma or for typing of undifferentiated carcinoma presents a challenge and in these cases, FNAC should be followed by excision biopsy.[11] The factors that influence the diagnostic specificity and sensitivity of FNAC in the diagnosis of lymphoma include necrosis in the involved nodes, the presence of dual pathology and sclerosis/fibrosis in involved nodes leading to insufficient diagnostic material.[12][13]

Among the four cases of Hodgkin's lymphoma, two were correctly diagnosed. Jimenez Hefferman J. A. et al in their studies had concluded that cytology offers a rapid and accurate approach not only for the diagnosis of recurrent Hodgkin's disease but also for its initial recognition. [14]

In our study, there were 17 cases of metastatic nodal diseases out of which 14 were correctly diagnosed by FNAC. Metastatic lesions comprised $13.9 \%$ of all cases and showed an accuracy of $89 \%$, sensitivity $80 \%$ and specificity $96 \%$. The metastatic tumours were metastasis from squamous cell carcinoma, adenocarcinoma, metastasis from carcinoma breast, metastasis from poorly differentiated carcinoma, small cell anaplastic carcinoma and carcinoma thyroid. Some morphological features helped to subtype these metastatic tumours and gave some directions to the identifications of 
possible primary site. Robert W. Astarita says that precise classification of metastatic neoplasms may prove difficult in less differentiated tumours especially if there is no nonprimary site.[15] FNAC is particularly useful for the documentation of metastatic carcinoma and can obviate further surgery performed merely to confirm the presence of metastasis.[1] The present study proves that FNAC is a useful tool in diagnosing metastasis with good certainty.

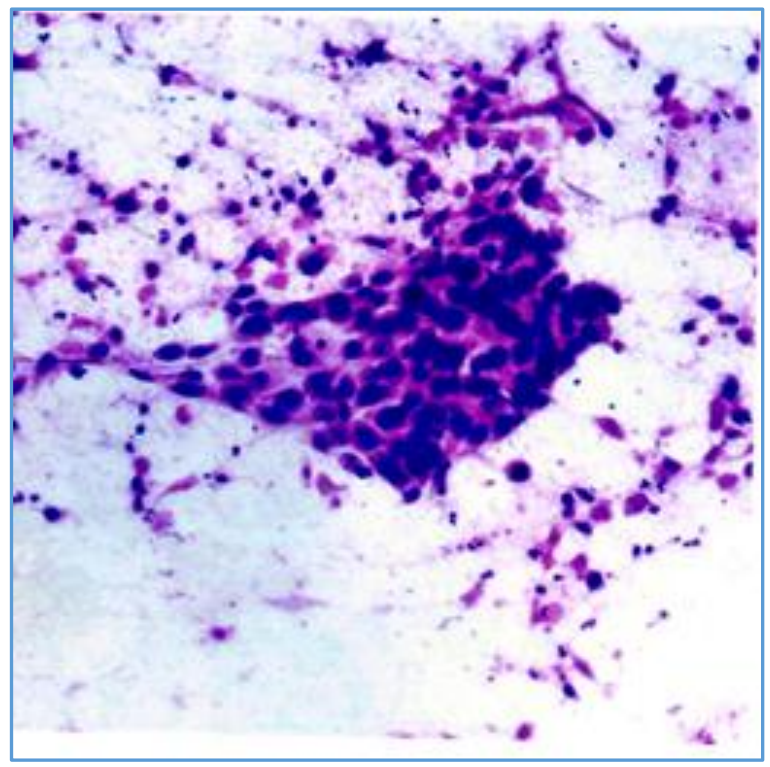

Figure 3. FNAC of Metastasis Lymph Node from Squamous Cell Carcinoma

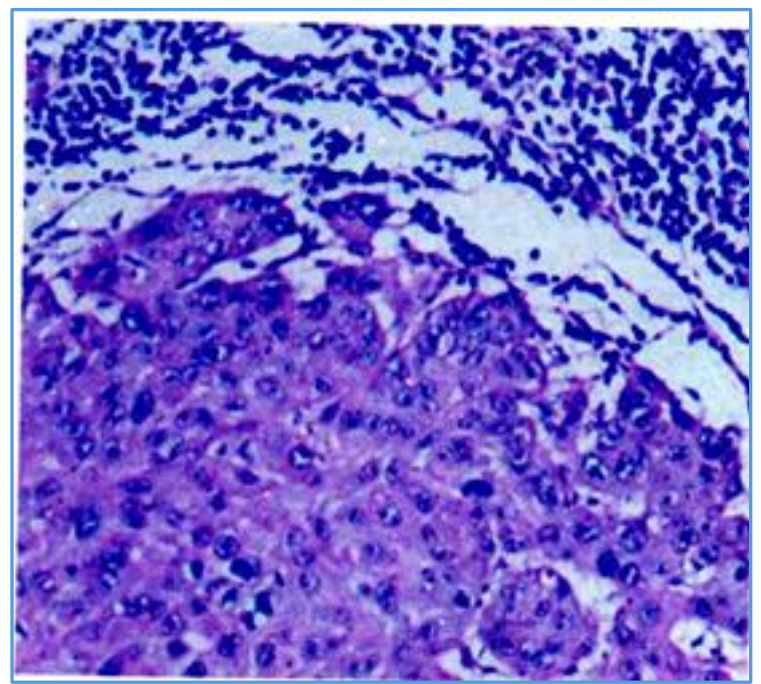

Figure 4. Histopathology of Metastasis Lymph Node from Squamous Cell Carcinoma

The improved diagnostic accuracy of FNAC in the diagnosis of malignant lesions of lymph nodes is probably due to a combination of factors such as availability of using and guidance of imaging technique as well as many other cytodiagnostic assistances like immunohistochemistry, trained and qualified manpower over the years.[16]

A total of 122 cases were studied with histological follow up in all cases. Out of 122 lymph node lesions 88 cases were correctly diagnosed. Sensitivity of the test was $84 \%$, specificity $95.8 \%$, positive predictive value $93.3 \%$, negative predictive value $89.6 \%$ and the overall diagnostic accuracy was $90.98 \%$.

Statistical Results of FNAC

\begin{tabular}{|c|c|}
\hline Sensitivity & $84 \%$ \\
\hline Specificity & $95.8 \%$ \\
\hline Positive predictive value & $93.3 \%$ \\
\hline Negative predictive value & $89.6 \%$ \\
\hline Percentage of false positive & $4.1 \%$ \\
\hline Percentage of false negative & $16 \%$ \\
\hline Overall accuracy & $90.98 \%$ \\
\hline
\end{tabular}

\section{CONCLUSION}

FNAC is an important diagnostic tool for diagnosing benign as well as malignant lesions. The overall accuracy of lymph node lesions by FNA was found to be $90.98 \%$. The overall accuracy of non-neoplastic lymph node lesions by FNAC was found to be $91.8 \%$, sensitivity $97.2 \%$ and specificity $84.3 \%$ whereas in neoplastic lymph node lesions the overall accuracy was $98.4 \%$, sensitivity $100 \%$ and specificity $97.5 \%$. Necrosis when seen with or without neutrophils, tuberculosis should be considered and a suppurative or other aetiology should be suggested only when AFB is negative and when smear shows large number of neutrophils. Findings in this study are comparable and consistent with studies conducted elsewhere. In the present study, it is observed that the accuracy of FNAC is marginally more in diagnosing neoplastic nodal diseases than non-neoplastic nodal diseases. In this context, it is to be ascertained that the improved diagnostic accuracy of FNAC in the diagnosis of malignant lesions of lymph nodes is probably due to a combination of factors such as availability of using and guidance of imaging technique as well as many other cytodiagnostic assistances like immunohistochemistry, trained and qualified manpower over the years.

\section{REFERENCES}

[1] Pandey P, Dixit A, Mahajan NC. The diagnostic value of FNAC in assessment of superficial palpable lymph nodes: a study of 395 cases. Al Ameen J Med Sci 2013;6(4):320-7.

[2] Guthrie CG. Gland puncture as a diagnostic measure. Johns Hopkins Hosp Bull 1921;32:266-9.

[3] Radhan RKS, Singh RP, Chaturvedi S. Place of fine needle aspiration cytology in the diagnosis of lymphadenopathy. Ind J Tub 1990;37:29.

[4] Saha I, Dasgupta S, Sen S. Spectrum of Cytopathological findings in cases of lymphadenopathy: a 3 years study. IOSR-JDMS 2016;15(2):59-63.

[5] Gupta SK, Dutta JK, Aikat M, et al. Evaluation of fine needle biopsy technique in diagnosis of tumours. Ind J Can 1975;12(3):257-67.

[6] Chandra K, Kumar P, Pandey S. Cytomorphological evaluation of lymphadenopathy on fine needle aspiration cytology -a hospital based retrospective study. JEMDS 2014;3(51):11929-37.

[7] Steel BL, Schwartz MR, Ramzy I. Fine needle aspiration biopsy in the diagnosis of lymphadenopathy in 1,103 patients. Role, limitations and analysis of diagnostic pitfalls. Acta Cytologica 1995;39(1):76-81. 
[8] Lapuerta P, Martin SE, Ellison E. Fine needle aspiration of peripheral lymph nodes in patients with tuberculosis and HIV. Am J Clin Pathol 1997; 107 (3): 317-20.

[9] Handa U, Palta A, Mohan $\mathrm{H}$, et al. Fine Needle aspiration diagnosis of tuberculosis lymphadenitis. Trop Doct 2002; 32 (3): 147-9.

[10] Mathur S, Kapila K, Dawar R, et al. Utility of fine needle aspiration cytology in the diagnosis and grading of lymphomas. Cyto Con 2003. 33rd Annual conference of Indian Academy of Cytology MR. Pune.

[11] Ahmed N, Israr S, Ashraf MS. Comparison of FNAC and excision biopsy in diagnosis of cervical lymphadenopathy. Pakistan Journal of Surgery 2009; 25(2): 72-5.

[12] Dong HY, Harris NL, Preffer FI, et al. Fine needle aspiration biopsy in the diagnosis and classification of primary and recurrent lymphoma: a retrospective analysis of the utility of cytomorphology and flow cytometry. Mod Pathol 2001;14(5):472-81.
[13] Morris-Stiff G, Cheang P, Key S, et al. Does the surgeon still have a role to play in the diagnosis and management of lymphomas? World J Surg Oncol 2008; 6: 13.

[14] Jimenez-Heffernan JA, Vicandi B, Lopez-Ferrer P, et al. Value of fine needle aspiration cytology in the initial diagnosis of Hodgkin's disease, Analysis of 188 cases with an emphasis on diagnostic pitfalls. Acta Cytol 2001; 45 (3):300-6.

[15] Astarita RW. Practical cytopathology. Churchill Livingstone 1990.

[16] Nama S, Majumder N, Nath S, et al. Cytodiagnostic spectrum of metastatic lymph nodes - a prospective study. JEMDS 2016;5(103):7554-8. 